\title{
Implementation Strategies for Workplace Data Collection: A Case Study
}

Leslie K. Erickson`, Frank J. Mierzwa ${ }^{\dagger}$, Sarah K. With ${ }^{\ddagger}$, Georgia T. Karuntzos ${ }^{\star \star}$, Kimberly E. Fox ${ }^{\dagger \dagger}$, Susan M. McHale ${ }^{\ddagger \ddagger}$, Orfeu M. Buxton $^{\star \star *}$

Tags: health measures, longitudinal, lay interviewer, logistics, in-person survey implementation, blood collection, workplace study DOI: $10.29115 /$ SP-2015-0026

\section{Survey Practice}

Vol. 8, Issue 5, 2015

In this paper, we describe the methods used for the successful implementation of a longitudinal survey in a workplace setting. Data for the Work, Family \& Health Study (WFHS) were collected at baseline and 6, 12, and 18 months post-baseline, and consisted of computer-assisted interviews, basic health measures, dried blood spot collection, and collection of sleep data via an actigraph watch. Data collection in the workplace presents unique logistical and operational challenges. Based on our experience, we discuss these challenges and offer key suggestions for successfully planning and implementing in-person data collection in a workplace setting.

\section{A CASE STUDY}

\section{INTRODUCTION}

We describe methods implemented to successfully plan, schedule, and conduct longitudinal in-person survey activities, including biological data collection with employees and their supervising managers in their workplace setting for the Work, Family, \& Health Study (WFHS). The study investigates how work conditions affect the health and wellbeing of employees and their families, with data collected at baseline, 6, 12, and 18 months post-baseline. Data were collected on company paid time within two distinct workplace contexts an information technology (IT) organization and an extended-care services organization. Trained lay field interviewers collected data through computer-assisted personal interviews (CAPI), basic health measures (height, weight, and blood pressure), blood collection (through both dried blood spots [DBS] and use of a point-of-care device to obtain immediate hemoglobin A1c [HbA1c] readings), and actigraph data obtained from wrist devices (Spectrum, Philips/Respironics) worn by participants to record sleep and wake behavior

\footnotetext{
* Institution: RTI International

† Institution: RTI International

$\ddagger$ Institution: RTI International

** Institution: RTI International

†† Institution: Bridgewater State University Department: Sociology

¥ Institution: Pennsylvania State University Department: Human Development and Family Studies Institution: Brigham and Women's Hospital; Pennsylvania State University; Harvard Medical School; Harvard T.H. Chan

*** School of Public Health
} 
for 7 days. Employees and managers were required to complete the interview with physical measures to be eligible for the DBS and actigraphy collection. Irrespective of the industry, the collection of survey and biological data at the workplace can present many unique operational challenges, including obtaining and maintaining buy-in from workplace leadership, securing and using work space for collection activities, recruiting participants, setting and completing appointments, collecting and processing biospecimens, and properly disposing of biohazard material. Special attention should be given to tailoring implementation strategies to the type of workplace and employee. Ultimately, the study was able to achieve very high response rates across all four waves of data collection, and across both industries.

\section{METHODS}

The core components of our data collection implementation strategy are described below and include (1) field staff training; (2) subject recruitment; (3) scheduling appointments; and (4) logistics.

\section{FIELD STAFF TRAINING}

The protocols are not difficult for lay interviewers to grasp, although longer training sessions are required. One interviewer is able to complete all data collection activities, which builds rapport and can reduce the burden on the participant by minimizing the number of visits needed to complete study activities. The minimally invasive biomarkers (height, weight, blood pressure, blood collection via finger-stick, and actigraphy) make this approach feasible and cost-effective.

All WFHS field staff attended an 8-day training session. An essential part of training involved hands-on practice using materials and equipment, conducting mock interviews, completing the health measures, practicing blood collection, and documenting activities. All WFHS field staff were required to pass certification on various aspects of the study before beginning fieldwork. Throughout the study, study staff also led in-person refresher trainings to review procedures or demonstrate new blood collection protocols on an as-needed basis.

\section{PARTICIPANT RECRUITMENT}

As described by Bray et al. (2013), the WFHS recruited employees and managers from each company to participate in the study. Company 1 (IT industry) utilized employee groups reporting to the same senior management team (roughly analogous to departments). Company 2 (extended-care industry) included 30 geographically distinct work sites distributed across six states.

Company 1 employees and managers were eligible if they were part of the selected work groups. Company 2 employees and managers were eligible if they worked at least 22.5 hours per week in direct patient care, and on the day or 
evening shifts.

Unlike in-home surveys, we needed to consider the organizational structure and different layers of individuals we had to engage as part of the participant recruitment process. We found developing working relationships with key personnel as early as possible was essential. Obtaining industry partner buy-in started with securing the support of top-level management, then communicating with managers and their direct-report employees. Through preliminary discussions with key management, we learned we needed to tailor and use differing approaches to communicating and sharing study information with participants in each industry. Within Company 1 , we shared information via web-postings and e-mail communication. Within Company 2, where employees did not have company e-mail addresses or mailboxes to receive hardcopy information, study information was provided via meet-and-greet sessions and as inserts with paychecks. We developed a suite of professional, high-quality printed materials tailored to each industry to convey the importance and legitimacy of the study.

We maintained a positive working relationship with each company throughout the life of the study by designating project staff as site managers. These individuals served as liaisons between study staff and company staff, ensured adherence to the company's regulations and culture, sustained communication between company management, site managers, and study staff, and worked with workplace representatives to identify approaches that best fit within the work structure and schedules to minimize disruption.

\section{SCHEDULING APPOINTMENTS}

Across workplace settings, we considered the stresses and demands on employees' work time when selecting a time to complete the survey. This included being flexible to meet employees' needs and being readily able to adjust appointment dates and times due to unexpected circumstances in the employees' workdays. We established two scheduling methods tailored to each industry.

Scheduling Appointments Within Company 1 Generally, Company 1 employees worked varied schedules and were required to work within tight deadlines. Many telecommuted, which required flexibility with when and where interview appointments were scheduled. To standardize the e-mail communication between field staff and selected employees and managers, the initial and follow-up e-mail messages were programmed as templates on the field staff laptops. Data collection took place in dedicated conference rooms to ensure privacy.

Scheduling Appointments Within Company 2 During the pilot study, we discovered that it could be extremely difficult for Company 2 employees to leave their patients for the entire time length required to complete all survey components in one session (75-90 minutes). So a critical protocol adaptation 
was to allow flexibility in breaking the appointment into separate visits when needed. Additionally, employees worked set shifts, scheduled by the shift supervisor, and they were unable to make their own decisions about an appropriate time to "come off the floor." To schedule an appointment, the field supervisor (FS) coordinated with the scheduler to develop an interview schedule. Every subject was assigned a time slot to meet with the field staff. The schedule often required tailoring as frequently as daily to accommodate unexpected circumstances (e.g., medical emergencies, staff absent from work).

\section{LOGISTICS}

Workspace Setup Proper interviewing space was arranged with the sites in advance of data collection. This was facilitated by the site manager, industry staff, and the FS. Our space requirements for conducting the CAPI interview included room to set up a digital scale and stadiometer, a flat surface for the field staff's laptop and showcard booklets, an electrical outlet (for the laptop), and sufficient privacy to guarantee confidentiality. In addition, certain parameters were required in choosing the space used to collect DBS. These included selecting an area not intended for eating, free of unrelated project equipment, with room for movement, and with privacy.

Company 1 was able to provide unused office space for our long-term use. But in Company 2, securing space of any kind was much more difficult. Interviewers worked anywhere from empty offices to the facility hair salon. All field staff were trained on how to properly set up the blood collection lab area and necessary precautions, similar to the guidelines outlined by Ostler et al. (2014), to make sure efficient and valid collections could take place.

Handling Biological Hazard Waste The blood spot collection produced biological hazard waste that required special handling and proper disposal. The project had to determine proper storage and disposal procedures in both industries. In Company 1, we were introducing biological hazard waste to an office environment that would not typically have blood or sharps on the premises. For proper storage and disposal, we purchased mail-back biomedical waste disposal kits that included all required materials to collect, store, package, and ship the biomedical waste for proper disposal.

In Company 2, the procedure for storing and disposing of biomedical waste was discussed as part of the initial meetings with each facility's staff. Because of the nature of the work in extended care facilities, procedures were already in place for biomedical waste disposal, and the project was able to dispose of waste on-site.

\section{RESULTS}

Across all waves of worksite data collection, we completed 9,194 CAPI interviews with physical measures, collected 7,794 blood samples, and completed actigraphy collection with 7,159 employees and managers. This 
resulted in unweighted response rates of 89.3 percent for the interview, 95.7 percent for the blood collection, and 87.9 percent for the actigraphy collection (using AAPOR $2011 \mathrm{RR} 2$ ). Listed in Table 1 are unweighted response rates (AAPOR 2011 RR2) by company for the different components of the survey process over the four waves of data collection, as indicators of the study's success at obtaining high cooperation at baseline and maintaining the cooperation during the follow-up waves.

Table 1 Unweighted response rates by wave.

\begin{tabular}{|c|c|c|c|c|}
\hline & Baseline & 6 months & 12 months & 18 months \\
\hline \multicolumn{5}{|c|}{ Interview with physical measures } \\
\hline IT company & $72.6 \%$ & $91.7 \%$ & $93.2 \%$ & $90.5 \%$ \\
\hline Extended-care company & $85.7 \%$ & $95.7 \%$ & $94.5 \%$ & $96.1 \%$ \\
\hline \multicolumn{5}{|l|}{ Dried blood spot collection } \\
\hline IT company & $92.6 \%$ & $93.9 \%$ & $94.3 \%$ & $95.4 \%$ \\
\hline Extended-care company & $94.4 \%$ & $97.2 \%$ & $97.8 \%$ & $98.2 \%$ \\
\hline \multicolumn{5}{|l|}{ Actigraphy collection } \\
\hline IT company & $87.1 \%$ & $86.6 \%$ & $84.7 \%$ & $85.0 \%$ \\
\hline Extended-care company & $88.9 \%$ & $87.4 \%$ & $88.4 \%$ & $91.8 \%$ \\
\hline
\end{tabular}

The baseline interview response rate for Company 1 was approximately 13 percent less than for Company 2, with interview response rates in subsequent waves exceeding 90 percent in both companies.

As shown in Table 1, the study achieved blood collection response rates in excess of 93 percent at baseline in both companies and maintained very high response rates in subsequent waves. The baseline response rates and the high response rates at follow-up are indicative of a highly energized and well-trained interviewer team and a high degree of trust and comfort by respondents with the interviewing staff completing the blood collection activities at the worksite.

Participants were asked to wear the actigraph watch for a full week. As shown in Table 1, actigraphy response rates at baseline were at 87 percent and 89 percent for Companies 1 and 2, respectively, and remained high across the four waves.

Another indicator of WFHS blood collection success is the quantity and integrity of the specimens collected and delivered to the laboratory for analysis. From the 7,794 cases with blood collected, field staff obtained $\mathrm{HbA} 1 \mathrm{c}$ readings using a point-of-service device (DCA Vantage Analyzer, Siemens Healthcare Diagnostics) on 99 percent of the cases. From the collected DBS cards, tests were run and results obtained over 98 percent of the time for all blood results of interest for the study: total cholesterol, high-density lipoprotein cholesterol, and C-reactive protein.

\section{DISCUSSION}

The implementation of in-person data collection activities in the workplace 
presents many logistical and operational challenges. Based on our experience, we offer the following key suggestions for successfully planning and implementing in-person data collection in a workplace setting:

- Flexibility, tailoring, and adaptability are of utmost importance in completing in-person data collection in the workplace. When interviewing employees at work, it is important to remember the increased burden being placed on them in the context of the workday. Although our study benefited from employees completing the interview on company paid time, in most instances, employees still had to make up the work they missed. In Company 1 , our data collection could add to an already stressful and busy schedule. In Company 2, study participation from direct-care employees meant planning for patient coverage during interviews and dealing with the unpredictability of shift work. Our study had to be cognizant of and address these issues up front when dealing with the workplace leadership and participants to gain their cooperation. Our approaches to data collection were further tailored to each industry per pilot study findings and adapted where needed across waves of collection. This included offering flexibility in scheduling, providing interviewing space in convenient locations, and proposing split appointments.

- Recruitment activities for in-person data collection in the workplace require proper planning and accommodation of many layers of personnel.

- Our recruitment started with top-level management, but continued with key, site-level personnel working directly with study participants. For working through data collection logistics and planning, an FS teamed with the site manager to work closely with the key personnel (which varied by industry) to obtain their buy-in and to make sure data collection activities at each wave started properly and ran smoothly.

- Participant recruitment was tailored to the industry, particularly the modes of communication for sharing information with the respondents.

\section{- Development and maintenance of strong working} relationships with the key personnel in each industry and the building and maintenance of participant trust and comfort with our field staff were key elements to our high cooperation rates across the data collection waves. In addition, the dedicated site managers for each industry allowed an ongoing presence at the workplaces that allowed continuation of relationships throughout the study. 
- Our study was able to build and maintain a high level of trust and comfort by:

- Using a very well-trained and professional research team, including interviewers, FSs, and site managers;

- Assigning field staff to the same participants for each wave, allowing them to build rapport over time;

- Expressing our recognition of the study's demands on participants' work time by being flexible with scheduling appointments and efficient with completing them; and

- Providing field staff with the skills and tools to guide their efficient setup, use, and breakdown of space provided for data collection activities.

To learn more about the Work, Family \& Health Study, please visit www.Work FamilyHealthNetwork.org.

\section{ACKNOWLEDGEMENTS}

This research was conducted as part of the Work, Family, \& Health Network ( www.WorkFamilyHealthNetwork.org), comprising eight research organisations conducting studies on how to improve the health of workers and their families and reduce work-family conflict, while also benefiting the organisations they work for. The study is funded by a cooperative agreement through the National Institutes of Health and the Centers for Disease Control and Prevention: Eunice Kennedy Shriver National Institute of Child Health and Human Development (Grant \# U01HD051217, U01HD051218, U01HD051256, U01HD051276), National Institute on Aging (Grant \# U01AG027669), the National Heart, Lung, and Blood Institute (R01HL107240), Office of Behavioral and Science Sciences Research, and National Institute for Occupational Safety and Health (Grant \# U01OH008788, U01HD059773). Grants from the William T. Grant Foundation, Alfred P. Sloan Foundation, and the Administration for Children and Families have provided additional funding. The contents of this publication are solely the responsibility of the authors and do not necessarily represent the official views of these institutes and offices. 


\section{REFERENCES}

American Association Public Opinion Research (AAPOR). 2011. Standard Definitions. Final Dispositions of Case, Codes and Outcome, Rates for Surveys. 7th ed. AAPOR.

Bray, J.W., E.L. Kelly, L.B. Hammer, D.M. Almeida, J.W. Dearing, R.B. King, and O.M. Buxton. 2013. An Integrative, Multilevel, and Transdisciplinary Research Approach to Challenges of Work, Family, and Health. RTI Press publication No. MR-0024-1303. Research Triangle Park, NC: RTI Press.

Ostler, M.W., J.H. Porter, and O.M. Buxton. 2014. "Dried Blood Spot Collection of Health Biomarkers to Maximize Participation in Population Studies.” J. Vis. Exp, no. 83 (January). https://doi.org/10.3791/50973. 